\title{
Mechanical aberrations in hypetrophic cardiomyopathy: emerging concepts
}

\author{
Dimitrios Ntelios ${ }^{1,2 *}$, Georgios Tzimagiorgis ${ }^{1}$, Georgios K. Efthimiadis ${ }^{2}$ and \\ Haralambos Karvounis $^{2}$
}

${ }^{1}$ Laboratory of Biological Chemistry, Medical School, Aristotle University of Thessaloniki, Thessaloniki, Greece, ${ }^{2}$ Department of Cardiology, AHEPA University Hospital, Thessaloniki, Greece

OPEN ACCESS

Edited by:

Mark T. Ziolo,

The Ohio State University, USA

Reviewed by:

Jonathan P. Davis,

The Ohio State University, USA

Jerry Curran,

The Ohio State University, USA

Mark J. Kohr

Johns Hopkins Bloomberg School of

Public Health, USA

*Correspondence:

Dimitrios Ntelios,

Laboratory of Biological Chemistry,

Medical School, Aristotle University of

Thessaloniki, University Campus,

54124 Thessaloniki, Greece

ntdimitris@yahoo.gr

Specialty section:

This article was submitted to

Clinical and Translational Physiology,

a section of the journal

Frontiers in Physiology

Received: 21 April 2015 Accepted: 03 August 2015

Published: 19 August 2015

Citation:

Ntelios D, Tzimagiorgis G, Efthimiadis GK and Karvounis H (2015) Mechanical aberrations in hypetrophic cardiomyopathy: emerging concepts.

Front. Physiol. 6:232

doi: 10.3389/fphys.2015.00232
Hypertrophic cardiomyopathy is the most common monogenic disorder in cardiology. Despite important advances in understanding disease pathogenesis, it is not clear how flaws in individual sarcomere components are responsible for the observed phenotype. The aim of this article is to provide a brief interpretative analysis of some currently proposed pathophysiological mechanisms of hypertrophic cardiomyopathy, with a special emphasis on alterations in the cardiac mechanical properties.

Keywords: hypertrophic cardiomyopathy, cardiac mechanics, stretch activation, epicardial-endocardial synergy, LVOT obstruction, myocardial disarray

\section{Introduction}

Hypertrophic cardiomyopathy (HCM) is a relatively rare inherited cardiovascular disease that affects approximately 1 in 500 young individuals and that accounts for $35 \%$ of sudden cardiac death cases in young athletes (Maron et al., 1995, 2009). It is characterized by a segmental hypertrophy of the left ventricle (LV) that mainly affects the interventricular septum, although there are no factors present that could have induced hypertrophy, such as hypertension or aortic stenosis. This type of hypertrophy has been termed asymmetrical, in contrast to the symmetrical hypertrophy in hypertensive patients, in which all LV segments are equally affected. Additional prominent features include left ventricular outflow tract (LVOT) obstruction, owing to an abnormal anterior motion of the mitral valve during systole that obstructs blood flow to the aorta, and disorganization of the myocardial tissue architecture (myocardial disarray). Some patients experience severe symptoms (dyspnea, palpitations, fainting, and sudden cardiac arrest caused by ventricular tachycardia), whereas others are asymptomatic. The onset is usually during adolescence but can occur later in life (Sherrid, 2006; Maron and Maron, 2013).

Regarding its genetic cause, myosin heavy chain beta (MYH7) and myosin binding protein $\mathrm{C}$ (MYBPC3) are the most commonly involved genes, followed by troponin I (TNNI3), troponin T (TNNT2), essential myosin light chain (MYL3), regulatory myosin light chain (MYL2), alpha tropomyosin (TPM1), and cardiac actin (ACTC) (Konno et al., 2010). These genes encode proteins of the sarcomere, which is the subcellular structure responsible for myocyte contraction. This paper explores how mutations in genes encoding sarcomeric proteins affect heart mechanical behavior and also focuses on unresolved issues regarding HCM disease pathomechanisms. 


\section{The Sarcomere}

Sarcomeres are composed of thick filaments consisting of the molecular motor myosin and thin filaments that are formed by actin (Figure 1A). Myosin is a protein complex of two heavy chains (MYH6 or MYH7), which are the main motor, as well as two regulatory (MYL2) and two essential light chains (MYL3) with a structural and regulatory role (Harris et al., 2011). Myosin exerts force on the thin filaments by ATP hydrolysis. Because of the action of myosin, the filaments slide over each other, leading to sarcomere contraction (Fatkin and Graham, 2002). The troponins (T, I, and C) and tropomyosin form a protein complex attached to the thin filaments, which regulates contraction in response to $\mathrm{Ca}^{2+}$. Upon excitation and $\mathrm{Ca}^{2+}$ entry into the myocardial cell, $\mathrm{Ca}^{2+}$ binds to troponin and enables actin-myosin interaction (Fatkin and Graham, 2002). Finally, myosin binding protein $\mathrm{C}$ is attached to the thick and thin filaments, modulates the myosin kinetics, and is a substrate of PKA and CAMKII phosphorylation (James and Robbins, 2011). With the exception of MYBPC, where HCM mutations lead to low protein levels (frameshift mutations and deletions), a gain-of-function mutation alters the mechanical properties of these proteins (ATPase rate, force generating capacity and sliding velocity for myosin, and $\mathrm{Ca}^{2+}$ affinity for troponin) (Harris et al., 2011; Moore et al., 2012; Spudich, 2014). Additionally, there are normal local variations in the function of some of these proteins across different segments of the normal heart with possible relevance to the pathophysiology of HCM.

\section{Normal Spatial Patterns in the Left Ventricle Mechanics}

The advent of newer imaging technologies has favored the detailed exploration of the regional function of the heart and the quantification of myocardial deformation (myocardial strain). Myocardial deformation analysis in the form of magnetic resonance imaging (MRI) has revealed strain dissimilarities between the endocardial and epicardial layers of the left ventricle (LV) wall and among different LV segments (Bogaert and Rademakers, 2001).

This lack of uniformity in contractility is also reflected on the molecular level. More specifically, the contraction capacity of the myosin heavy chain can be either fast (MYH6) or slow (MYH7). There are certain developmental, environmental, epigenetic and hormonal factors (e.g., thyroid hormones) that regulate the expression of MYH6 and $\mathrm{MYH} 7$, which are encoded by a gene cluster on chromosome 14 (Gupta, 2007). MYH7 is the predominant motor in the human ventricle. MYH7 has the same force-generating capacity as MYH6 but a lower ATPase rate (reduced energy cost). Moreover, MYH6 seems to be the predominant motor in cases in which fast kinetics are required, such as in the human atrium, the contraction time of which is shorter than that of the ventricles, or in mouse ventricles, where the heart rate is as fast as 500 beats per second (Gupta, 2007). Cardiac hypertrophy and heart failure causes MYH6 downregulation (Gupta, 2007). The importance of MYH6, despite the low level of MYH6 expression in human ventricles, is also highlighted in other studies that have observed an increase in MYH6 expression in heart failure patients who improved after cardiac resynchronization therapy or medical treatment (Lowes et al., 2002; Vanderheyden et al., 2008).

Several studies have examined the mechanical properties of the epicardial and endocardial layers (Cazorla et al., 2000, 2005). For example, Stelzer et al. report that there is a transmural gradient across the porcine left ventricular wall with epicardial myocytes comprising 13\% MYH6 and endocardial myocytes 3\% (Figure 1D) (Stelzer et al., 2008). Another study found that the presence of MYH6, even at low levels (10\%), speeds up the onset of mechanical contraction (Locher et al., 2011). Thus, subtle differences in mechanical properties exist between endocardial and epicardial layers.

In addition to the transmural heterogeneity, there is also an MYL2 phosphorylation gradient toward the apex in the murine heart (Figure 1D) (Davis et al., 2001). This post-translational modification, which increases the force and speed of contraction, favors contractility in the LV apex relating to the base (Bogaert and Rademakers, 2001; Toepfer et al., 2013). On the contrary, in the papillary muscles and the adjacent endocardial layer, there is an MYL2 hypophosphorylation (Figure 1D), leading to increased amplitude and rate of onset of the stretch-activation response (Figure 1B). In fact, the stretch activation response was shown to be critical for papillary muscle function (Vemuri et al., 1999).

\section{Mechanical Flaws in HCM Patients}

The spatial patterns and control mechanisms described above are compromised in HCM patients.

\section{Myosin Properties}

Normally, a myocardial cell has two options: to express either the slow MYH7 or the fast MYH6. The HCM mutations in the MYH7 gene generally increase the myosin velocity and ATPase rate (Moore et al., 2012; Spudich, 2014). Therefore, in the presence of a dominant MYH7 mutation, the cell produces a combination of wild type slow MYH7 (normal allele) and a fast mutated MYH7. Eventually, it becomes difficult for the cell to determine the operational characteristics of the myosin that it produces. This defect may prevent the heart from utilizing certain molecular strategies to fine-tune its regional mechanical properties (e.g., transmural MYH6 gradients), thus leading to the altered pattern of ventricular contraction (ventricular dyssynchrony) that is detected in HCM patients (Chen et al., 2012). Furthermore, the increased ATPase activity in conjunction with the increased volume of the hypertrophied cell (low surface to volume ratio, increased anabolic needs) push the energy production system of the cell to its limits (Moore et al., 2012). For instance, Crilley et al. conducted a magnetic resonance spectroscopy study in which they found that even the mutation carriers that did not present any hypertrophy had a reduced phosphocreatine to ATP ratio (Crilley et al., 2003). In fact, this reduction in energy reserves was not confined to 
A

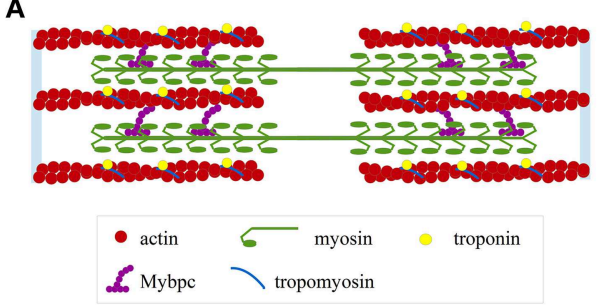

C

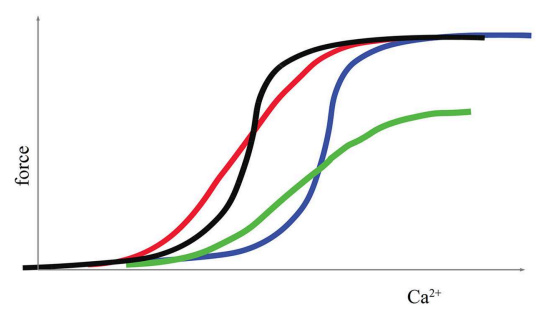

FIGURE 1 | Normal cardiac mechanics. (A) Graphical representation of the sarcomere (B) length-dependent activation: when a myocardial fiber is subjected to stretch there is an initial passive tension (brown curve) followed, with a time delay (blue arrow), by active force development (green curve). (C) $\mathrm{Ca}^{2+}$-force
B

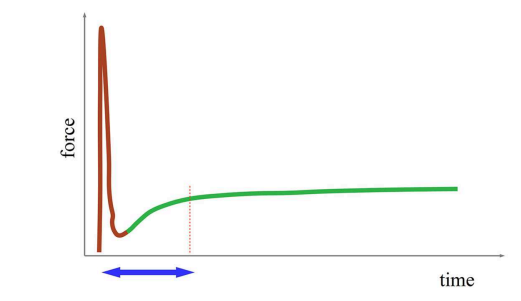

D

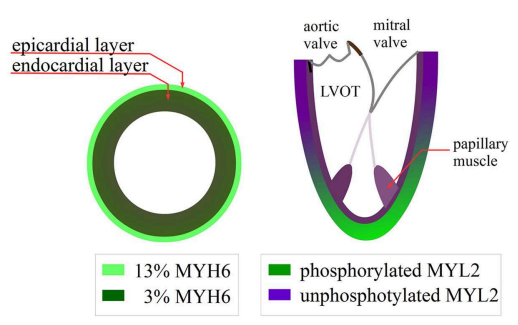

curve modulation by shifting the curve along the $x$ axis (changing myofilament affinity to $\mathrm{Ca}^{2+}$, black and blue curve), by changing its steepness (more graded response to $\mathrm{Ca}^{2+}$, red curve) and by changing maximal force (green curve) (D) MYH6 expression and MYL2 phosphorylation. only the MYH7 mutation carriers (Witjas-Paalberends et al., 2014).

\section{Stretch Activation Response and Calcium}

Another important control mechanism that closely relates to the Frank-Starling law and is defective in HCM patients is lengthdependent activation, which manifests as an increased force generation after myocardial stretching caused by an alteration in myofilament sensitivity to calcium (Figure 1B) (Campbell and Chandra, 2006; de Tombe et al., 2010; Sequeira et al., 2013). Myocardial cells collected from patients subjected to surgical myectomy had a defective response to stretch in vitro irrespective of the mutated gene (Sequeira et al., 2013). During exercise, HCM patients failed to increase stroke volume in part due to this defect (Critoph et al., 2014). An MYL3 HCM mutation in a mouse study affected the frequency range at which the heart can operate efficiently due to alteration of the stretch-activation response (Vemuri et al., 1999).

Additionally, several in vitro studies showed that in HCM there was a calcium ion mishandling due to reduced sarcoplasmic reticulum $\mathrm{Ca}^{2+}$ ATPase, alteration in $\mathrm{Na}^{+} / \mathrm{Ca}^{2+}$ cotransporter function, $\mathrm{t}$ tubule reduction, increased $\mathrm{Ca}^{2+}$ in the sarcoplasmic reticulum, and possibly low energy reserves, given that $\mathrm{Ca}^{2+}$ pumping is a process that consumes a large amount of ATP (Coppini et al., 2013; Lan et al., 2013). In addition, mutations in MYH7, MYBPC3, TNNI3, and TPM1 increased the myofilament calcium affinity and reduced the calcium-force curve steepness (Figure 1C) (i.e., a more graded response to calcium input) (van Dijk et al., 2012; Sequeira et al., 2013; Ramirez-Correa et al., 2014). A slower rate of tension development in response to a calcium influx reduces the energy efficiency because, for the same force, an increase in $\mathrm{Ca}^{2+}$ concentration is needed and consequently an increase in ATP consumption for $\mathrm{Ca}^{2+}$ recycling (Sun and Irving, 2010).

\section{Epicardial-endocardial Synergy and Disease-specific Features}

The orientation and the sequence of activation of myocardial fibers are of paramount importance for the mechanical function of the heart. The fiber orientation across the LV wall changes from $60^{\circ}$ to $-60^{\circ}$ from the endocardium to the epicardium (Figure 2A). During the initial phase of ventricular systole and before aortic valve opening (isovolumetric contraction), there is an activation of mainly endocardial fibers. The contraction of the endocardial fibers stretches the epicardial fibers, which is an important step for the subsequent mechanical activation of the epicardial fibers during the ejection phase (Ashikaga et al., 2009). As described above, the defect in the stretch activation response may compromise the epicardial fiber contraction, thus posing an extra mechanical load on the endocardial fibers, leading to their hypertrophy. Further evidence from an MRI study of the hypertrophy distribution shows that that hypertrophy typically starts in the anteroseptal region and spirals in a counterclockwise manner toward the apex (Figure 2B) (Florian et al., 2012). According to the Torrent Guasp myocardial band model, the hypertrophied segment corresponds to the so-called descending helix (endocardial fibers) (Buckberg et al., 2008; Sengupta and Narula, 2012). Likewise, a myocardial deformation analysis, in which MRI was used, revealed that the endocardial fibers were contracting properly, whereas the epicardium was hypocontractile with non-contracting or even stretched segments (Aletras et al., 2011). Whether the hypokinesis of the epicardial 

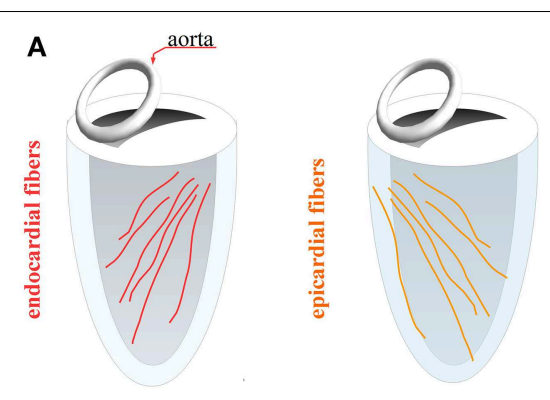

C
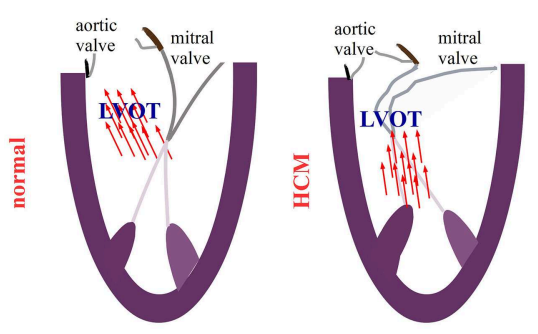

FIGURE 2 | LV mechanics in HCM. (A) Endocardial fibers (red) and epicardial fibers (orange) orientation (B) distribution of hypertrophied areas in the left ventricle (LV) wall (spiral pattern) (C) left ventricular outflow tract

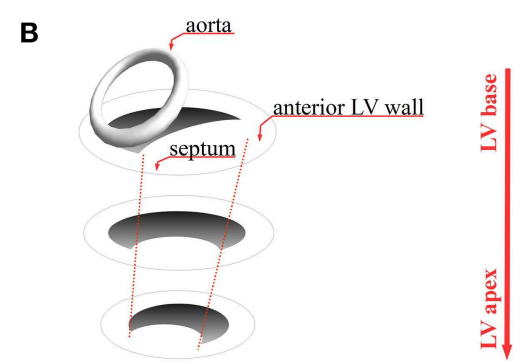

D

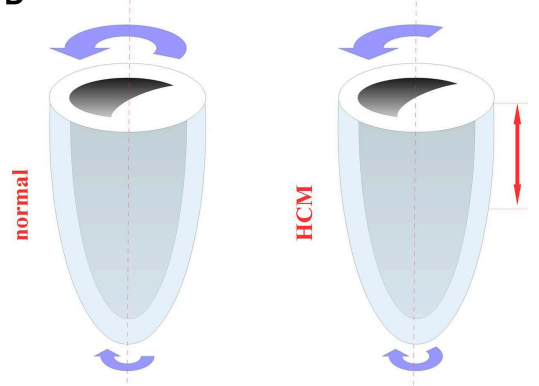

fibers in patients may facilitate the emergence of myocardial disarray in this area, much like the disarray observed in detached papillary muscles after mitral valve replacement surgery, needs to be explored (Pirolo et al., 1982). Indeed, a human MRI study showed myocardial disarray in hypokinetic areas (Tseng et al., 2006). Conversely, in an HCM mouse model (MyBPC knockout), the MRI revealed an increased disarray in the endocardial side, although this finding was not histologically confirmed (Wang et al., 2010).

There is increasing evidence that this abnormal coordination between the epicardial and the endocardial fibers may relate to LVOT obstruction. The heart orchestrates the contraction of different segments to achieve decreased flow turbulence and handle energy losses (Sengupta et al., 2012). Furthermore, the coordinated movements of the epicardial and endocardial fibers during isovolumetric contraction and relaxation are crucial for the proper flow redirection between the outflow and the inflow tract (Sengupta et al., 2007). For example, when alterations occur in the heart contraction pattern, because of the epicardial pacing from the left ventricle base, there are also observable effects on the intracavitary flow pattern (Goetz et al., 2005; Sengupta et al., 2007). In patients with LVOT obstruction, there was a misdirection of flow toward the outflow tract during ejection. In particular, the flow affected the posterior aspect of the mitral valve, causing its anterior displacement, thus obstructing the outflow tract (Figure 2C) (Ro et al., 2014). In HCM, the left ventricle adopted an abnormal contraction pattern, characterized by an increased deformation in the circumferential direction and a decreased deformation in the longitudinal (base to apex) direction. Additionally, the increased circumferential strain in obstructive vs. non-obstructive HCM may contribute to LVOT obstruction (Carasso et al., 2008; Ntelios et al., 2015). Concerning the alleviation of the LVOT obstruction in some patients after implantation of a pacemaker, pacing from the right ventricle apex may alter the LV contraction pattern and redirect flow in an advantageous way (Sherrid, 2006). Another factor that facilitates LVOT obstruction is the elongation of the mitral valve leaflets (Ro et al., 2014). This defect may be secondary to the abnormal intracavitary flow pattern because the hydrodynamic conditions are of paramount importance for proper valve formation during the developmental stages (Kalogirou et al., 2014).

Owing to the geometrical configuration of the epicardial and endocardial fibers, during systole, the left ventricle performs a wringing motion (Figure 2D). This motion is quantitatively assessed as a "ventricular twist" (the difference of rotation between the base and the apex). The physiological role of this ventricular twist is to homogenize the wall stress across the ventricular wall, thus increasing the involvement of the epicardial fibers during contraction (Sengupta et al., 2008). Carasso et al. observed that in HCM patients the net ventricular twist did not differ from the control group (Carasso et al., 2008). Nonetheless, the rotation of the midventricular part, relating to the left ventricle base, was minimal, and the apical part was the main contributor to the total angle of rotation (Figure 2D) (Carasso et al., 2008). Therefore, this locally reduced twist in the base and midventricular part may be an additional factor increasing the mechanical load of the endocardial fibers, leading to their hypertrophy. In fact, HCM patients failed to increase the ventricular twist during exercise (Soullier et al., 2012). Another important consideration is that the ventricular twist 
depends on geometrical factors, such as the ventricular length and diameter (Young and Cowan, 2012), which can be modified in a hypertrophied heart.

\section{Apical Hypertrophy}

In some cases, hypertrophy predominately involves the LV apex (Chen et al., 2011). Some studies have shown that HCM patients present a blunted base to apex gradient in contractility and have reduced apical rotation and stretched apical segments (Reddy et al., 2008; Chang et al., 2010). These characteristics highlight the reduced systolic function of the apex in these patients. Theoretically, defects in the MYL2 and other unrecognized mechanisms that enhance apical contraction might lead to compensatory apical hypertrophy. Additionally, aneurysm formation is an anticipated complication given the increased apical wall stress (Guccione et al., 1995; Chen et al., 2011). Furthermore, mutations in the MYL2 and MYL3 genes are connected to the midcavitary hypertrophy variant (Poetter et al., 1996). In this HCM subtype, there is hypertrophy of the midventricular part of the LV wall and papillary muscles possibly because MYL3 and MYL2 can modulate the amplitude and rate of onset of the stretch-activation response, thus compromising papillary muscle function (Poetter et al., 1996; Vemuri et al., 1999; Arad et al., 2005; Stelzer et al., 2006). Another important clinical observation is that patients with the same mutation present an apical or a more classical asymmetrical hypertrophy pattern (Arad et al., 2005). Additionally, 10\% of HCM patients present apical hypertrophy with concurrent septal hypertrophy (Florian et al., 2012). Thus, the different phenotypes in these patients may simply reflect the variable extent of hypertrophy toward the base.

\section{References}

Aletras, A. H., Tilak, G. S., Hsu, L. Y., and Arai, A. E. (2011). Heterogeneity of intramural function in hypertrophic cardiomyopathy: mechanistic insights from MRI late gadolinium enhancement and high-resolution DENSE strain maps. Circ. Cardiovasc. Imaging 4, 425-434. doi: 10.1161/CIRCIMAGING.110.958751

Arad, M., Penas-Lado, M., Monserrat, L., Maron, B. J., Sherrid, M., Ho, C. Y., et al. (2005). Gene mutations in apical hypertrophic cardiomyopathy. Circulation 112, 2805-2811. doi: 10.1161/CIRCULATIONAHA.105. 547448

Ashikaga, H., van der Spoel, T. I., Coppola, B. A., and Omens, J. H. (2009). Transmural myocardial mechanics during isovolumetric contraction. JACC Cardiovasc. Imaging 2, 202-211. doi: 10.1016/j.jcmg.2008. 11.009

Bogaert, J., and Rademakers, F. E. (2001). Regional nonuniformity of normal adult human left ventricle. Am. J. Physiol. Heart Circ. Physiol. 280, H610-H620.

Buckberg, G., Hoffman, J. I., Mahajan, A., Saleh, S., and Coghlan, C. (2008). Cardiac mechanics revisited: the relationship of cardiac architecture to ventricular function. Circulation 118, 2571-2587. doi: 10.1161/CIRCULATIONAHA.107.754424

Campbell, K. B., and Chandra, M. (2006). Functions of stretch activation in heart muscle. J. Gen. Physiol. 127, 89-94. doi: 10.1085/jgp.200509483

Carasso, S., Yang, H., Woo, A., Vannan, M. A., Jamorski, M., Wigle, E. D., et al. (2008). Systolic myocardial mechanics in hypertrophic cardiomyopathy: novel concepts and implications for clinical status. J. Am. Soc. Echocardiogr. 21, 675-683. doi: 10.1016/j.echo.2007.10.021

\section{Treatment Options in HCM}

The treatment of HCM has so far largely focused on treating the symptoms of the disease using beta adrenergic receptor blockers. B-blockers slow the heart rate and reduce myocardial oxygen consumption and LVOT obstruction. Disopyramide and the calcium channel blocker verapamil are also helpful. In cases of drug-refractory symptoms, alleviation of outflow tract obstruction by either surgical septal myectomy or percutaneous infusion of alcohol in the septal branch of the left coronary artery is the current practice. A drug-based approach targeting length-dependent activation, myofilament $\mathrm{Ca}^{2+}$ affinity and myosin properties is very promising. Another important issue in the management of these patients is risk stratification for sudden cardiac death (SCD). In general terms, patients with unexplained syncope, documented ventricular tachycardia or aborted SCD, positive family history for SCD, maximal LV wall thickness $>30 \mathrm{~mm}$ and abnormal drop in blood pressure during exercise, treated with an implantable defibrillator (Maron and Maron, 2013; Efthimiadis et al., 2014).

\section{Conclusion}

Hypertrophic cardiomyopathy exhibits significant heterogeneity (i.e., numerous mutations and a variable clinical course). The mechanisms analyzed in this paper shed some light on the relation of mutations to disease phenotype, although they may not necessarily apply to all patients. A better understanding of the pathophysiological mechanisms leading to hypertrophy will contribute to the further improvement of treatment and to risk stratification for sudden cardiac death.

Cazorla, O., Le Guennec, J. Y., and White, E. (2000). Lenght-tension relationships of subepicardial and subendocardial single ventricular myocytes from rat and ferret hearts. J. Mol. Cell Cardiol. 32, 735-744. doi: 10.1006/jmcc.2000.1115

Cazorla, O., Szilagyi, S., Le Guennec, J. Y., Vassort, G., and Lacampagne, A. (2005). Transmural strechdependent regulation of contractile properties in rat heart and its alteration after myocardial infraction. FASEB J. 19, 88-90. doi: 10.1096/fj.04-2066fje

Chang, S. A., Kim, H. K., Kim, D. H., Kim, J. C., Kim, Y. J., Kim, H. C., et al. (2010). Left ventricular twist mechanics in patients with apical hypertrophic cardiomyopathy: assessment with 2D speckle tracking echocardiography. Heart 96, 49-55. doi: 10.1136/hrt.2009.166629

Chen, C. C., Lei, M. H., Hsu, Y. C., Chung, S. L., and Sung, Y. J. (2011). Apical hypertrophic cardiomyopathy: correlations between echocardiographic parameters, angiographic left ventricular morphology, and clinical outcomes. Clin. Cardiol. 34, 233-238. doi: 10.1002/clc.20874

Chen, J., Nagaraj, H., Bhambhani, P., Kliner, D. E., Soman, P., Garcia, E. V., et al. (2012). Effect of alcohol septal ablation in patients with hypertrophic cardiomyopathy on left-ventricular mechanical dyssynchrony as assessed by phase analysis of gated SPECT myocardial perfusion imaging. Int. J. Cardiovasc. Imaging 28, 1375-1384. doi: 10.1007/s10554-011-9942-y

Coppini, R., Ferrantini, C., Yao, L., Fan, P., Del Lungo, M., Stillitano, F., et al. (2013). Late sodium current inhibition reverses electromechanical dysfunction in human hypertrophic cardiomyopathy. Circulation 127, 575-584. doi: 10.1161/CIRCULATIONAHA.112.134932

Crilley, J. G., Boehm, E. A., Blair, E., Rajagopalan, B., Blamire, A. M., Styles, P., et al. (2003). Hypertrophic cardiomyopathy due to sarcomeric gene mutations is characterized by impaired energy metabolism irrespective of the degree 
of hypertrophy. J. Am. Coll. Cardiol. 41, 1776-1782. doi: 10.1016/S07351097(02)03009-7

Critoph, C. H., Patel, V., Mist, B., and Elliott, P.M. (2014). Cardiac output response and peripheral oxygen extraction during exercise among symptomatic hypertrophic cardiomyopathy patients with and without left ventricular outflow tract obstruction. Heart 100, 639-646. doi: 10.1136/heartjnl-2013304914

Davis, J. S., Hassanzadeh, S., Winitsky, S., Lin, H., Satorius, C., Vemuri, R., et al. (2001). The overall pattern of cardiac contraction depends on a spatial gradient of myosin regulatory light chain phosphorylation. Cell 107, 631-641. doi: 10.1016/S0092-8674(01)00586-4

de Tombe, P. P., Mateja, R.D., Tachampa, K., Ait Mou, Y., Farman, G. P., and Irving, T. C. (2010). Myofilament length dependent activation. J. Mol. Cell. Cardiol. 48, 851-858. doi: 10.1016/j.yjmcc.2009.12.017

Efthimiadis, G. K., Pagourelias, E. D., Gossios, T., and Zegkos, T. (2014). Hypertrophic cardiomyopathy in 2013: current speculations and future perspectives. World J. Cardiol. 6, 26-37. doi: 10.4330/wjc.v6.i2.26

Fatkin, D., and Graham, R. M. (2002). Molecular mechanisms of inherited cardiomyopathies. Physiol. Rev. 82, 945-980. doi: 10.1152/physrev.00012.2002

Florian, A., Masci, P. G., De Buck, S., Aquaro, G. D., Claus, P., Todiere, G., et al. (2012). Geometric assessment of asymmetric septal hypertrophic cardiomyopathy by CMR. JACC Cardiovasc. Imaging 5, 702-711. doi: 10.1016/j.jcmg.2012.03.011

Goetz, W. A., Lansac, E., Lim, H. S., Weber, P. A., and Duran, C. M. (2005). Left ventricular endocardial longitudinal and transverse changes during isovolumic contraction and relaxation: a challenge. Am. J. Physiol. Heart Circ. Physiol. 289, H196-H201. doi: 10.1152/ajpheart.00867.2004

Guccione, J. M., Costa, K. D., and McCulloch, A. D. (1995). Finite element stress analysis of left ventricular mechanics in the beating dog heart. J. Biomech. 28, 1167-1177. doi: 10.1016/0021-9290(94)00174-3

Gupta, M. P. (2007). Factors controlling cadiac myosin-isoform shift during hypertrophy and heart failure. J. Mol. Cell. Cardiol. 43, 388-403. doi: 10.1016/j.yjmcc.2007.07.045

Harris, S. P., Lyons, R. G., and Bezold, K. L. (2011). In the thick of it: HCMcausing mutations in myosin binding proteins of the thick filament. Circ. Res. 108, 751-764. doi: 10.1161/CIRCRESAHA.110.231670

James, J., and Robbins, J. (2011). Signaling and Myosin-binding Protein C. J. Biol. Chem. 286, 9913-9919. doi: 10.1074/jbc.R110.171801

Kalogirou, S., Malissovas, N., Moro, E., Argenton, F., Stainier, D. Y., and Beis, D. (2014). Intracardiac flow dynamics regulate atrioventricular valve morphogenesis. Cardiovasc. Res. 104, 49-60. doi: 10.1093/cvr/cvu186

Konno, T., Chang, S., Seidman, J. G., and Seidman, C. E. (2010). Genetics of hypertrophic cardiomyopathy. Curr. Opin. Cardiol. 25, 205-209. doi: 10.1097/HCO.0b013e3283375698

Lan, F., Lee, A. S., Liang, P., Sanchez-Freire, V., Nguyen, P. K., Wang, L., et al. (2013). Abnormal calcium handling properties underlie familial hypertrophic cardiomyopathy pathology in patient-specific induced pluripotent stem cells. Cell. Stem Cell 12, 101-113. doi: 10.1016/j.stem.2012.10.010

Locher, M. R., Razumova, M. V., Stelzer, J. E., Norman, H. S., and Moss, R. L. (2011). Effects of low-level $\alpha$-myosin heavy chain expression on contractile kinetics in pocrine myocardium. Am. J. Physiol. Heart Circ. Physiol. 300, H869-H878. doi: 10.1152/ajpheart.00452.2010

Lowes, B. D., Gilbert, E. M., Abraham, W. T., Minobe, W.A., Larrabee, P., Ferguson, D., et al. (2002). Myocardial gene expression in dilated cardiomyopathy treated with beta-blocking agents. N. Engl. J. Med. 346, 1357-1365. doi: 10.1056/NEJMoa012630

Maron, B. J., Doerer, J. J., Haas, T. S., Tierney, D. M., and Mueller, F.O. (2009). Analysis of 1866 Deaths in the United States, 1980-2006. Circulation 119, 1085-1092. doi: 10.1161/CIRCULATIONAHA.108.804617

Maron, B. J., Gardin, J. M., Flack, J. M., Gidding, S. S., Kurosaki, T. T., and Bild, D. E. (1995). Prevalence of hypertrophic cardiomyopathy in a general population of young adults: echocardiographic analysis of 4111 subjects in the CARDIA Study. Circulation 92, 785-789. doi: 10.1161/01.CIR.92.4.785

Maron, B. J., and Maron, M. S. (2013). Hypertrophic cardiomyopathy. Lancet 381, 242-255. doi: 10.1016/S0140-6736(12)60397-3

Moore, J. R., Leinwand, L., and Warshaw, D. M. (2012). Understanding cardiomyopathy phenotypes based on functional impact of mutations in the myosin motor. Circ. Res. 111, 375-385. doi: 10.1161/CIRCRESAHA.110.223842
Ntelios, D., and Efthimiadis, G., Tzimagiorgis, G. (2015). The strain, the valve and the LVOT obstruction. J. Am. Coll. Cardiol. 12, 2050. doi: 10.1016/j.jacc.2015.01.057

Pirolo, J. S., Hutchins, G. M., Moore, G. W., and Weisfeldt, M. L. (1982). Myocyte disarray develops in papillary muscles released from normal tension after mitral valve replacement. Circulation 66, 841-846. doi: 10.1161/01.CIR.66.4.841

Poetter, K., Jiang, H., Hassanzadeh, S., Master, S. R., Chang, A., Dalakas, M. C., et al. (1996). Mutations in either the essential or regulatory light chains of myosin are associated with a rare myopathy in human heart and skeletal muscle. Nat. Genet. 13, 63-69. doi: 10.1038/ng0596-63

Ramirez-Correa, G. A., Frazier, A. H., Zhu, G., Zhang, P., Rappold, T., Kooij, V., et al. (2014). Cardiac Troponin I Pro82Ser variant induces diastolic dysfunction, Blunts $\beta$-Adrenergic response and impairs myofilament cooperativity. J. Appl. Physiol. 118, 212-223. doi: 10.1152/japplphysiol.00463.2014

Reddy, M., Thatai, D., Bernal, J., Pradhan, J., and Afonso, L. (2008). Apical hypertrophic cardiomyopathy: potential utility of strain imaging. Eur. J. Echocardiogr. 9, 560-562. doi: 10.1016/j.euje.2007.02.004

Ro, R., Halpern, D., Sahn, D. J., Homel, P., Arabadjian, M., Lopresto, C., et al. (2014). Vector flow mapping in obstructive hypertrophic cardiomyopathy to assess the relationship of early systolic left ventricular flow and the mitral valve. J. Am. Coll. Cardiol. 64, 1996-1999. doi: 10.1016/j.jacc.2014.04.090

Sengupta, P. P., Khandheria, B. K., Korinek, J., Jahangir, A., Yoshifuku, S., Milosevic, I., et al. (2007). Left ventricular isovolumetric flow sequence during sinus and paced rhythms: new insights from use of high-resolution Doppler and ultrasonic digital particle imaging velocimetry. J. Am. Coll. Cardiol. 49, 899-908. doi: 10.1016/j.jacc.2006.07.075

Sengupta, P. P., and Narula, J. (2012). LV segmentation and mechanics in HCM: twisting the Rubik's Cube into perfection! JACC Cardiovasc. Imaging 5, 765-768. doi: 10.1016/j.jcmg.2012.05.009

Sengupta, P. P., Pedrizzetti, G., Kilner, P. J., Kheradvar, A., Ebbers, T., Tonti, G., et al. (2012). Emerging trends in CV flow visualization. JACC Cardiovasc. Imaging 5, 305-316. doi: 10.1016/j.jcmg.2012.01.003

Sengupta, P. P., Tajik, A. J., Chandrasekaran, K., and Khandheria, B. K. (2008). Twist mechanics of the left ventricle: principles and application. JACC Cardiovasc. Imaging 1, 366-376. doi: 10.1016/j.jcmg.2008.02.006

Sequeira, V., Wijnker, P. J., Nijenkamp, L. L., Kuster, D. W., Najafi, A., WitjasPaalberends, E. R., et al. (2013). Perturbed length-dependent activation in human hypertrophic cardiomyopathy with missense sarcomeric gene mutations. Circ. Res.112, 1491-1505. doi: 10.1161/CIRCRESAHA.111.300436

Sherrid, M. V. (2006). Pathophysiology and treatment of hypertrophic cardiomyopathy. Prog. Cardiovasc. Dis. 49, 123-151. doi: 10.1016/j.pcad.2006.08.001

Soullier, C., Obert, P., Doucende, G., Nottin, S., Cade, S., Perez Martin, A., et al. (2012). Exercise response in hypertrophic cardiomyopathy: blunted left ventricular deformational and twisting reserve with altered systolic-diastolic coupling. Circ. Cardiovasc. Imaging 5, 324-332. doi: 10.1161/CIRCIMAGING.111.968859

Spudich, J. A. (2014). Hypertrophic and dilated cardiomyopathy: four decades of basic research on muscle lead to potential therapeutic approaches to these devastating genetic diseases. Biophys. J. 106, 1236-1249. doi: 10.1016/j.bpj.2014.02.011

Stelzer, J. E., Norman, H. S., Chen, P. P., Patel, J. R., and Moss, R. L. (2008). Transmural variation in myosin heavy chain isoform expression modulates the timing of myocardial force generation in porcine left ventricle. J. Physiol. 586, 5203-5214. doi: 10.1113/jphysiol.2008.160390

Stelzer, J. E., Patel, J. R., and Moss, R. L. (2006). Acceleration of stretch activation in murine myocardium due to phosphorylation of myosin regulatory light chain. J. Gen. Physiol. 128, 261-272. doi: 10.1085/jgp.200609547

Sun, Y. B., and Irving, M. (2010). The molecular basis of the steep forcecalcium relation in heart muscle. J. Mol. Cell Cardiol. 48, 859-865. doi: 10.1016/j.yjmcc.2009.11.019

Toepfer, C., Caorsi, V., Kampourakis, T., Sikkel, M. B, West, T. G., Leung, M. C., et al. (2013). Myosin regulatory light chain (RLC) phosphorylation change as a modulator of cardiac muscle contraction in disease. J. Biol. Chem. 288, 13446-13454. doi: 10.1074/jbc.M113.455444

Tseng, W. Y., Dou, J., Reese, T. G., and Wedeen, V. J. (2006). Imaging myocardial fiber disarray and intramural strain hypokinesis in hypertrophic 
cardiomyopathy with MRI. J. Magn. Reson. Imaging 23, 1-8. doi: 10.1002/jmri.20473

Vanderheyden, M., Mullens, W., Delrue, L., Goethals, M., de Bruyne, B., Wijns, W., et al. (2008). Myocardial gene expression in heart failure patients treated with cardiac resynchronization therapy: responders versus non responders. J. Am. Coll. Cardiol. 51, 129-136. doi: 10.1016/j.jacc.2007.07.087

van Dijk, S. J., Paalberends, E. R., Najafi, A., Michels, M., Sadayappan, S., Carrier, L., et al. (2012). Contractile dysfunction irrespective of the mutant protein in human hypertrophic cardiomyopathy with normal systolic function. Circ. Heart Fail. 5, 36-46. doi: 10.1161/CIRCHEARTFAILURE.111. 963702

Vemuri, R., Lankford, E. B., Poetter, K., Hassanzadeh, S., Takeda, K., Yu, Z. X., et al. (1999). The stretch-activation response may be critical to the proper functioning of the mammalian heart. Proc. Natl. Acad. Sci. U.S.A. 96, 1048-1053. doi: 10.1073/pnas.96.3.1048

Wang, T. T., Kwon, H. S., Dai, G., Wang, R., Mijailovich, S. M., Moss, R. L., et al. (2010). Resolving myoarchitectural disarray in the mouse ventricular wall with diffusion spectrum magnetic resonance imaging. Ann. Biomed. Eng. 38, 2841-2850. doi: 10.1007/s10439-010-0031-5
Witjas-Paalberends, E. R., Güçlü, A., Germans, T., Knaapen, P., Harms, H. J., Vermeer, A. M., et al. (2014). Gene-specific increase in the energetic cost of contraction in hypertrophic cardiomyopathy caused by thick filament mutations. Cardiovasc. Res. 103, 248-257. doi: 10.1093/cvr/cvu127

Young, A. A., and Cowan, B. R. (2012). Evaluation of left ventricular torsion by cardiovascular magnetic resonance. J. Cardiovasc. Magn. Reson. 14:49. doi: $10.1186 / 1532-429 \mathrm{X}-14-49$

Conflict of Interest Statement: The authors declare that the research was conducted in the absence of any commercial or financial relationships that could be construed as a potential conflict of interest.

Copyright (C) 2015 Ntelios, Tzimagiorgis, Efthimiadis and Karvounis. This is an openaccess article distributed under the terms of the Creative Commons Attribution License (CC BY). The use, distribution or reproduction in other forums is permitted, provided the original author(s) or licensor are credited and that the original publication in this journal is cited, in accordance with accepted academic practice. No use, distribution or reproduction is permitted which does not comply with these terms. 